\title{
SETM*-MaxK: An Efficient SET-Based Approach to Find the Largest Itemset
}

\author{
Ye-In Chang and Yu-Ming Hsieh \\ Dept. of Computer Science and Engineering \\ National Sun Yat-Sen University \\ Kaohsiung, Taiwan, Republic of China \\ Tel: 886-7-5252000 (ext. 4334), Fax: 886-7-5254301 \\ changyi@cse.nsysu.edu.tw
}

\begin{abstract}
In this paper, we propose the SETM*-MaxK algorithm to find the largest itemset based on a high-level set-based approach, where a large itemset is a set of items appearing in a sufficient number of transactions. The advantage of the set-based approach, like the SETM algorithm, is simple and stable over the range of parameter values. In the SETM*-MaxK algorithm, we efficiently find the $L_{k}$ based on $L_{w}$, where $L_{k}$ denotes the set of large $k$-itemsets with minimum support, $L_{k} \neq \emptyset, L_{k+1}=\emptyset$ and $w=2^{\left\lceil\log _{2} k\right\rceil-1}$, instead of step by step. From our simulation, we show that the proposed SETM*-MaxK algorithm requires shorter time to achieve its goal than the SETM algorithm.
\end{abstract}

\section{Introduction}

One of the important data mining tasks, mining association rules in transactional or relational databases, has recently attracted a lot of attention in database communities 125 . The task is to discover the important associations among items such that the presence of some items in a transaction will imply the presence of other items in the same transaction [3]. Previous approaches to mining association rules can be classified into two approaches: low-level and high-level approaches, where a low-level approach means to retrieve one tuple from the relational database at a time, and a high-level approach means a set-based approach. For example, Apriori/AprioriTID [1] and DHP [5] are based on the low-level approach, while the SETM algorithm [4 is based on the high-level approach. A set-based approach (i.e., a high-level approach) allows a clear expression of what needs to be done as opposed to specifying exactly how the operations are carried out in a low-level approach. The declarative nature of this approach allows consideration of a variety of ways to optimize the required operations. Eventually, it should be possible to integrate rule discovery completely with the database system. This would facilitate the use of the large amounts of data that are currently stored on relational databases. The relational query optimizer can then determine the most efficient way to obtain the desired results. Finally, the set-based approach has a small number of well-defined, simple 
concepts and operations. This allows easy extensibility to handling additional kinds of mining, e.g. relating association rules to customer classes.

In [4, based on a high-level approach, Houtsma and Swami proposed the SETM algorithm that uses SQL for generating the frequent itemsets. Algorithm SETM is simple and stable over the range of parameter values. Moreover, it is easily parallelized. But the disadvantage of the SETM algorithm is that it generates too many invalid candidate itemsets. In this paper, we design the SETM*-MaxK algorithm to find the largest itemset based on a high-level setoriented approach. One of the applications to find the largest itemset is that in a grocery store, we may want to know the maximum set of data items which will be bought in one transaction by the most of customers. In the SETM*-MaxK algorithm, we efficiently find the $L_{k}$ based on $L_{w}$, where $L_{k}$ denotes the set of large $k$-itemsets with minimum support, $L_{k} \neq \emptyset, L_{k+1}=\emptyset$ and $w=2^{\left\lceil\log _{2} k\right\rceil-1}$, instead of step by step. From our simulation, we show that the proposed SETM*MaxK algorithm needs shorter time to achieve its goal than the SETM algorithm.

\section{The SETM*-MaxK Algorithm}

Sometimes, we may only want to know the maximum set of data items which will be bought in one transaction by the most of customers. That is, we only want to find out the maximum $k$ such that $L_{k} \neq \emptyset$ and $L_{k+1}=\emptyset$. In this Section, we present the $S E T M^{*}-M a x K$ algorithm to achieve such a goal. In the proposed algorithm, we make use of the "jump" approach and the binary search approach to efficiently find the maximum $k$. We use the "jump" approach to efficiently construct $L_{k}$ based on $L_{w}$, until $L_{k}=\emptyset$, where $w=k / 2$.

Table 1. Variables used in the $S E T M^{*}-M a x K$ algorithm

\begin{tabular}{|c|l|}
\hline$R_{k}^{\prime}$ & A database of candidate $k$-itemsets(i.e., a candidate DB) \\
\hline$L_{k}$ & Large $k$-itemsets \\
\hline$C_{k}$ & Candidate $k$-itemsets \\
\hline$R_{k}$ & A database of large $k$-itemsets(i.e., a filtered DB) \\
\hline half_k & Last $k$ processed \\
\hline eq_len & Record the length of the same items in the join step \\
\hline
\end{tabular}

Table 1 shows the variables used in the $S E T M^{*}-M a x K$ algorithm. The complete algorithm are shown in Figures 2, In procedure $S E T M^{*}-M a x K$, the first step (i.e., the forward phase) is to generate $R_{k}^{\prime}, L_{k}$, and $R_{k}$, except $R_{1}^{\prime}$, until $L_{k}=\emptyset$ or $C_{k}=\emptyset$, where $k=2^{i}, i \geq 0$. To generate counts for those patterns in $R_{k}^{\prime}$ that meet the minimum support constraint, we call procedure gen-Litemset. Before we go on to generate patterns of length $k+1$, we first have to select the tuples from $R_{k}^{\prime}$ that should be extended; that is, those tuples that meet the minimum support constraint. We also wish the resulting relation to be sorted 
on $R_{k}\left(\right.$ trans_id, item ${ }_{1}, \ldots$, item $\left.m_{k}\right)$. This can be done by calling procedure filter$D B$. After $L_{1}$ and $R_{1}$ are constructed, we then apply the "jump" approach. We repeat calling procedures gen-2k-C, gen-2k-CDB, gen-Litemset and filter-DB to generate $C_{k}, R_{k}^{\prime}, L_{k}$ and $R_{k}$, respectively, until $L_{k}=\emptyset$ or $C_{k}=\emptyset$, where $k \geq 2$. In procedure gen-2k-C, we construct $C_{k}$ based on $L_{h a l f_{-} k}$, where half_k $=k$ div 2. In procedure gen-2k-CDB, we could generate all lexicographically ordered patterns of length $k$ stored on $R_{k}^{\prime}\left(\right.$ trans_id, item ${ }_{1}, \ldots$, item $\left.m_{k}\right)$ based on $R_{h a l f_{-} k}$ only. Note that The SETM algorithm constructs $R_{k}^{\prime}$ based on $R_{k-1}$ and the original database $S A L E S$. Due to this reason, the SETM algorithm generates and counts too many candidates itemsets. To reduce the size of the candidate database $R_{k}^{\prime}$, we have a new strategy to construct $R_{k}^{\prime}$ in procedure gen-2k-CDB. Moreover, to avoid unnecessary construction of $R_{k}^{\prime}, L_{k}$ and $R_{k}$, we will first construct $C_{k}$ before we construct $L_{k}$. That is, $R_{k}^{\prime}, L_{k}$ and $R_{k}$ will be generated only if $C_{k} \neq \emptyset$.

In step 2 of procedure $S E T M^{*}-M a x K$, we will keep changing the value of target $K$ by considering the range between $k$ and hal $f_{\_} k(=k / 2)$, until MaxK 0 . We apply a variant (marked with $* *$ ) of the binary search, in which when $L_{\text {target } K}=\emptyset$, in additional to updating $H K$, we will update $L K=L K+1$ and compute $R_{L K}^{\prime}, L_{L K}$ and $R_{L K}$ if $C_{L K} \neq \emptyset$. In this way, for the next loop, $R_{\text {target } K}^{\prime}$ can be generated based on a new $R_{L K}$. For the example as shown in Figure 1 1 (a), Figure 1. (b) shows the process of procedure $S E T M^{*}-M a x K$ (as shown in Figure 21), where the minimum support $=3$.

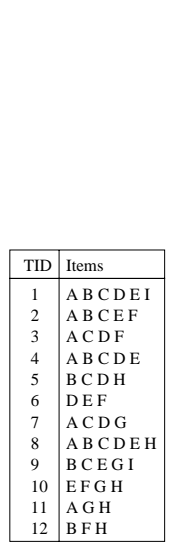

(a)
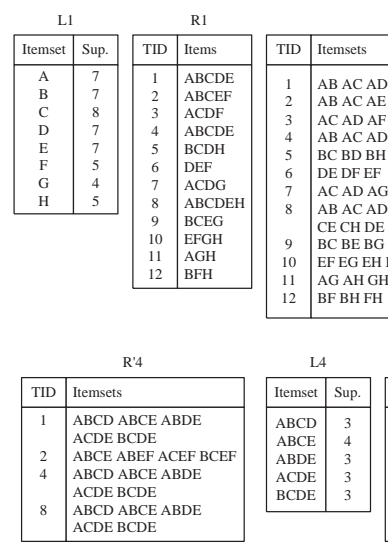

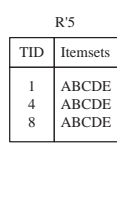

(b)

Fig. 1. (a) An example transaction database ; (b) The process of the SETM*MaxK algorithm 


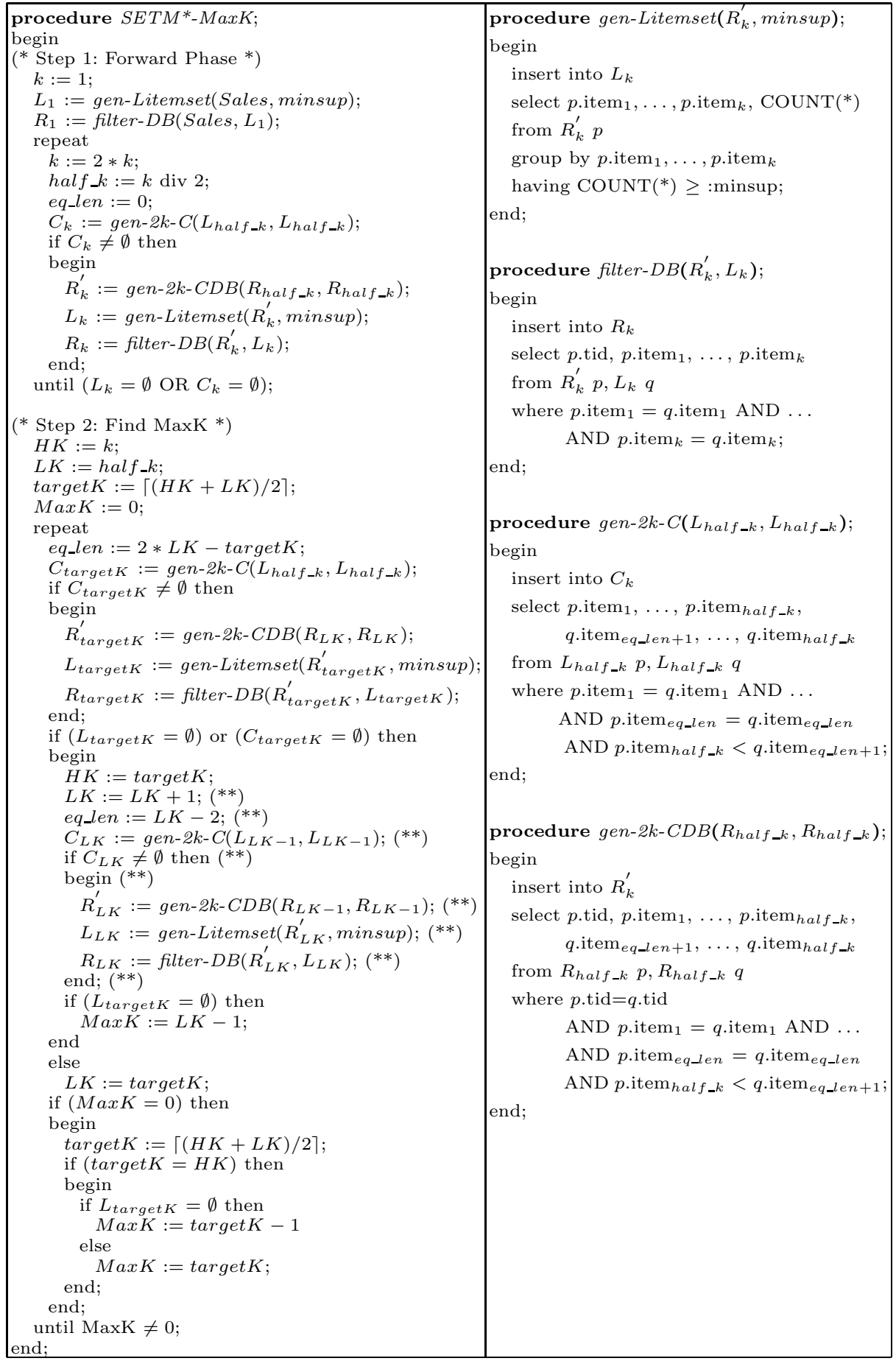

Fig. 2. The $S E T M^{*}$-MaxK procedure 


\section{Performance}

In this Section, we study the performance of the proposed SETM*-MaxK algorithm, and make a comparison with the SETM [4] algorithm by simulation. Our experiments were performed on a PentiumIII Server with one CPU clock rate of $450 \mathrm{MHz}, 128 \mathrm{MB}$ of main memory, running Windows-NT 2000, and coded in Delphi. The data resided in the Delphi relational database and was stored on a local 8G IDE 3.5" drive. Table 2 shows the parameters. The length of an

Table 2. Parameters

\begin{tabular}{|c|l|}
\hline$|D|$ & Number of transactions \\
$|T|$ & Average size of transactions \\
$|M T|$ & Maximum size of the transactions \\
$|I|$ & Average size of maximal potentially large itemsets \\
$|M I|$ & Maximum size of the potentially large itemsets \\
$|L|$ & Number of maximal potentially large itemsets \\
$N$ & Number of items \\
\hline
\end{tabular}

itemset in $\mathcal{F}$ (potentially maximal large itemsets) is determined according to a Poisson distribution with mean $\mu$ equal to $|I|$. The size of each potentially large itemset is between 1 and $|M I|$. Items in the first itemset are chosen randomly from the set of items. To model the phenomenon that large itemsets often have common items, some fraction of items in subsequent itemsets are chosen from the previous itemset generated. We use an exponentially distributed random variable with mean equal to the correlation level to decide this fraction for each itemset. The remaining items are picked at random. In the datasets used in the experiments, the correlation level was set to 0.5 . Each itemset in $\mathcal{F}$ has an associated weight that determines the probability that this itemset will be picked. The weight is picked from an exponential distribution with mean equal to 1 . The weights are normalized such that the sum of all weights equals 1 . For example, suppose the number of large itemsets is 5. According to the exponential distribution with mean equal to 1 , the probabilities for those 5 itemsets with ID equal to $1,2,3,4$ and 5 are $0.43,0.26,0.16,0.1$ and 0.05 , respectively, after the normalization process. These probabilities are then accumulated such that each size falls in a range. For each transaction, we generate a random real number which is between 0 and 1 to determine the ID of the potentially large itemset. To model the phenomenon that all the items in a large itemset are not always bought together, we assign each itemset in $\mathcal{F}$ a corruption level $c$. When adding an itemset to a transaction, we keep dropping an item from the itemset as long as a uniformly distributed random number (between 0 and 1) is less than $c$. The corruption level for an itemset is fixed and is obtained from a normal distribution with mean $=0.5$ and variance $=0.1$. Each transaction is stored in a file system with the form of $<$ transaction identifier, item $>$. Let Case 1 denotes $|T|=5,|M T|=10,|I|=4,|M I|=8,|D|=20 \mathrm{~K}$, Size $=1.5 \mathrm{MB}$, $N=1,000$ and $|L|=2,000$. When we choose Case 1 the synthetic dataset and with a minimum support $=0.33 \%$, the detailed information about $\left|R_{k}^{\prime}\right|,\left|L_{k}\right|$, 
$\left|R_{k}\right|$ and the execution time in these two algorithms are shown in Tables 3 (a), and 3 -(b). From these tables, similarly, we observe that the execution time of SETM*-MaxK algorithm is shorter than that of the SETM algorithm.

\section{Conclusion}

Discovery of association rules is an important problem in the area of data mining. In order to benefit from the experience with relational databases, a set-oriented approach to mining data is needed. In this paper, to find a large itemset of a specific size in relational database, we have efficiently found the $L_{k}$ based on $L_{w}$, where $L_{k} \neq \emptyset, L_{k+1}=\emptyset$ and $w=2^{\left\lceil\log _{2} k\right\rceil-1}$, instead of step by step. From our simulation results, we have shown that the proposed SETM*-MaxK algorithm requires shorter time to achieve their goals than the SETM algorithm.

Table 3. A comparison of storage space and execution time (Case 1): (a) SETM; (b) SETM*-MaxK.

\begin{tabular}{|c|c|c|c|c|c|c|c|c|c|c|c|c|c|c|}
\hline & \multicolumn{3}{|c|}{$L_{1}$} & \multicolumn{4}{|c|}{$L_{2}$} & \multicolumn{4}{|c|}{$L_{3}$} & \multicolumn{3}{|c|}{$L_{4}$} \\
\hline & $R_{1}^{\prime}$ & $L_{1}$ & $R_{1}$ & $R_{2}^{\prime}$ & $L_{2}$ & & $R_{2}$ & & $R_{3}$ & $L_{3}$ & $R_{3}$ & $R_{4}$ & $L_{4}$ & $R_{4}$ \\
\hline \multirow[t]{4}{*}{ SETM } & 116799 & 209 & - & 323149 & 714 & & 30093 & & 8789 & 384 & 50955 & 49139 & 121 & 11068 \\
\hline & & & & \multicolumn{3}{|c|}{$L_{5}$} & \multicolumn{3}{|c|}{$L_{6}$} & \multirow{2}{*}{\multicolumn{2}{|c|}{$\begin{array}{c}\text { Total Time } \\
\text { (seconds) }\end{array}$}} & & & \\
\hline & & & & $R_{5}^{\prime}$ & $L_{5}$ & $R_{5}$ & $R_{6}^{\prime}$ & $L_{6}$ & $R_{6}$ & & & & & \\
\hline & & & $\mathrm{ETN}$ & 17938 & 2 & 147 & 10 & 0 & 0 & & 2.45 & & & \\
\hline
\end{tabular}

(a)

\begin{tabular}{|c|c|c|c|c|c|c|c|c|c|c|c|c|}
\hline \multicolumn{10}{|c|}{$L_{1}$} & \multicolumn{3}{c|}{$L_{2}$} \\
\hline$R_{1}^{\prime}$ & $L_{1}$ & $R_{1}$ & $R_{2}^{\prime}$ & $L_{2}$ & $R_{2}$ & $R_{4}^{\prime}$ & $L_{4}$ & $R_{4}$ & $R_{5}^{\prime}$ & $L_{5}$ & $R_{5}$ & (seconds) \\
\hline 116799 & 209 & 113491 & 306160 & 714 & 130093 & 96673 & 121 & 10068 & 2464 & 2 & 147 & 46.63 \\
\hline
\end{tabular}

\section{References}

1. R. Agrawal and R. Srikant, "Fast Algorithms for Mining Association Rules in Large Databases," Proc. 20th Int'l Conf. Very Large Data Bases, pp. 490-501, Sept. 1994.

2. F. Berzal, J. Cubero, N. Marin, and J Serrano, "TBAR: An Efficient Method for Association Rule Mining in Relational Databases," Data and Knowledge Engineering, Vol. 37, No. 1, pp. 47-64, April 2001.

3. M.-S. Chen, J. Han, and P.S. Yu, "Data Mining: An Overview from a Database Perspective," IEEE Trans. on Knowledge and Data Engineering, Vol. 8, No. 5, pp. 866-882, Dec. 1996.

4. M. Houtsma and A. Swami, "Set-oriented Mining for Association Rules in Relational Databases," Proc. 11th IEEE Int'l Conf. Data Engineering, pp. 25-33, 1995.

5. J.-S. Park, M.-S. Chen, and P.S. Yu, "Using a Hash-Based Method with Transaction Trimming for Mining Association Rules," IEEE Trans. on Knowledge and Data Engineering, Vol. 9, No. 5, pp. 813-825, Sept. 1997.

6. S. Sarawagi, S. Thomas, and R. Agrawal, "Integrating Association Rule Mining with Relational Database Systems: Alternatives and Implications," Proc. 1998 ACM SIGMOD Int'l Conf. Management of Data, pp. 343-354, 1998. 